\title{
Quality and Value in Old Age Psychiatry John Wattis
}

Susan Mary Benbow, The community clinic: its advantages and disadvantages, International Journal of Geriatric Psychiatry, 5 (1990), I I 9-I 2 I.

Barry W. Rovner, Jane Lucas-Blanstein, Marshall F. Folstein and Shannon W. Smith, Stability over one year in patients admitted to a nursing home dementia unit. International fournal of Geriatric Psychiatry, 5 ( 1990$)$, 77-82.

Both these papers evaluate aspects of service delivery relevant to psychiatrically-ill people. The first extends the practice of home assessment by the psychiatrist to home follow-up. Psychiatrists in the United Kingdom have already started to run neighbourhood clinics, usually in general practitioners' surgeries, and Susan Benbow has taken the next logical step. She discontinued a conventional outpatient clinic and replaced it with a home-visiting scheme. The catchment area was divided into four zones which were visited in rotation over a four-week period. A different old people's home was also visited each week in the cycle. New patients and those requiring 'intensive' (more than monthly?) outpatient follow-up were excluded. The first six 'community clinics' were compared with the previous six full conventional clinics. Patients to be visited at home were not forewarned of the date of the follow-up visit unless they specifically asked to be. All patients were seen by the consultant with re-visits if the patient was out initially. In the community clinic, 44 of the 46 listed patients $(96 \%)$ were successfully seen as compared to 37 of the $49(75 \%)$ in the traditional clinic. In addition, 27 patients' carers were seen in the community clinic compared with nine in the traditional clinic. There was no significant difference between the patients in the number who had blood taken. Much of the non-attendance in the traditional sample appears to have been due to ambulance problems, for of those coming by their own transport $90 \%$ arrived, against $72 \%$ of these coming by ambulance. The distance travelled to the community clinic ranged from 7 to I I miles with a mean of 9.3 miles and a mean travel time of 2.6 hours. The author concluded that home visits provided a better quality service on the simple measures used as well as providing the clinician with a much fuller picture of the patients' function and home conditions.

Of course, many questions were left unasked and unanswered : for example, how many of the visits needed to be performed by a consultant as opposed to a junior doctor or a nurse? Nevertheless this 
simple paper is a landmark in examining quality and value issues in everyday practice. The sting in the tail is that the Department of Health does not collect data on 'informal' home visits so that this community workload is invisible in their indicators.

The second paper is from the United States where the private health care 'market' is already well established. The authors point out that the majority of the 1.5 million elderly Americans in nursing homes have mental or behavioural disturbances. After a discussion of the financial disincentives to research into the mental health of nursing home residents in the United States, the authors describe their own study of 2 I specially designated 'dementia unit' beds in a $\mathrm{I} 8 \mathrm{I}$ bed nursing home in Baltimore. Patients in the unit with a clinical diagnosis of Alzheimer's disease were compared with a control group of Alzheimer's disease patients in the same nursing home but not in the unit.

A multi-disciplinary term provided extra care for the dementia unit patients on an individualized basis. Dementia unit patients received 2.9 hours per day of nursing care, 40 hours per week of activities programme and weekly medical and psychiatric evaluation. Non-unit patients received 2.I hours each day of nursing care, $3-5$ hours per week of activities programme, physician evaluation every 30 days and no regular psychiatric care. Altogether 14 patients and 14 controls were evaluated. At the baseline unit patients were younger and received fewer medications than non-unit patients. Otherwise the groups were comparable to gender, level of care needed, severity of dementia and severity of behavioural problems. A year later, two unit patients ( $14 \%$ ) and nine non-unit patients $(64 \%)$ had declined in terms of level of care needed. Unfortunately cognitive assessments were not repeated.

The age-difference between the unit and non-unit patients, the differences in baseline level of medication and other problems in study design mean the results must be interpreted with some caution, but note that the first of these factors should logically have produced more rapid decline in the unit group. The sting in the tail of this paper is that the dementia unit group all paid higher daily rates for their care. In a private system only relatively rich people will be able to pay for the best quality care. In an 'internal market' with a restricted budget it will be very hard to implement indicators of quality of care sufficiently powerful to overcome political pressures to keep costs down.

St James's University Hospital, Leeds 